\title{
Greedy Forwarding for Mobile Social Networks Embedded in Hyperbolic Spaces
}

\author{
Jingwei Zhang \\ Department of Computer Science, Columbia University. \\ iceboal@gmail.com
}

\begin{abstract}
In this work, we design and evaluate a novel greedy forwarding algorithm using metrics in hyperbolic spaces. Hyperbolic geometry has a natural topological reflection of scale-free networks, and greedy algorithm failed in Euclidean space becomes possible in hyperbolic one. We show that mobile social networks can be successfully embedded in such spaces, and obtains competitive performance in terms of message delivery ratio and cost. Under this result, we thus intuitively reveal the fundamental reason that why the famous BUBBLE Rap achieves the optimal performance.
\end{abstract}

\section{Categories and Subject Descriptors}

C.2.0 [Computer-Communication Networks]: General; C.4 [Performance of Systems]: [Modeling techniques, Performance attributes]

\section{Keywords}

Greedy forwarding, mobile social networks, hyperbolic spaces

\section{INTRODUCTION}

We evidence a world with a great amount of portable devices carried by people forming the mobile social networks[1, 2 ]. In this work, we propose a message forwarding method in such environment by mapping the users into a metric space and then deploying greedy forwarding algorithm. In order to design forwarding mechanisms that enhance the message delivery possibility in a limited time interval without wasting network resources, one should learn from users' contact history. Several studies developed forwarding methods based on social and temporal factors gained by contact history, such as $[3,4]$.

We focus on an alternative approach using greedy forwarding algorithm with nodes mapped into 2D hyperbolic space as geographic metrics. Distance between nodes represents contact probabilities in the future. Then greedy algorithm is implemented by choosing next hop which maximize the distance to the destination [5]. This simple but robust method only requires devices to store location information,

Permission to make digital or hard copies of part or all of this work for personal or classroom use is granted without fee provided that copies are not made or distributed for profit or commercial advantage and that copies bear this notice and the full citation on the first page. Copyrights for thirdparty components of this work must be honored. For all other uses, contact the owner/author(s)

Copyright is held by the owner/author(s).

SIGCOMM'13, August 12-16, 2013, Hong Kong, China.

ACM 978-1-4503-2056-6/13/08.

http://dx.doi.org/10.1145/2486001.2491728.
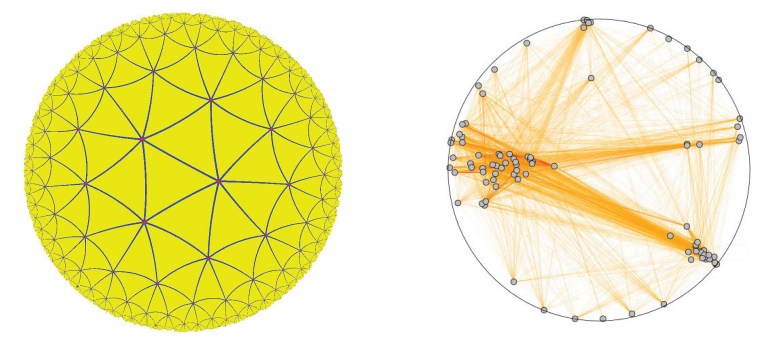

Figure 1: Hyperbolic Space

Figure 2: Mapping results of Reality Trace

meanwhile still achieves high message delivery ratio. The fundamental reason makes this method work is the relationship between hyperbolic space and scale-free networks. We know that even low-dimensional hyperbolic spaces are akin to infinite-dimensional Euclidean ones. Fig. 1 is an example of the hyperbolic plane in Poincare disk model, the edges in the figure are actually the same length in hyperbolic space.

If ignoring the transient period and treat user contacts as encounter processes, we can form a probability graph that the coefficient $p_{i j}$ of edge joins node $i, j$ represents the contact probability between $i, j$, and $\sum_{j} p_{i j}$ represents the expected degree of node $i$. Such a graph is similar with regular scale-free graph. Therefore, it is possible to implement this mapping method as a metric of greedy forwarding algorithm in mobile social networks.

In BUBBLE Rap[3], they designed social-based forwarding algorithms that utilizes community and centrality as metrics and obtained high delivery performance. Meanwhile, there is no theoretical analysis of why this greedy method works. We will show in Section 3 that our forwarding mechanism behaves almost the same as BUBBLE Rap. That is to say, since our greedy method has been proved successfully working in hyperbolic spaces theoretically [6], BUBBLE Rap is also proved to be close to optimal solutions.

\section{METHOD}

\subsection{Hyperbolic model}

Boguñá et al.[6] proposed a method to synthesize network with any desired number of nodes $N$, average degree $\bar{k}$, average clustering $\bar{C}$ and power-law distribution exponent $\gamma \geq 2$ by distributing $N$ nodes uniformly (under the hyperbolic metric) within a hyperbolic disc and link them with probability which decreases with the hyperbolic distance between them. Following this approach, we first distribute angular coordinates uniformly in $[0,2 \pi)$ and distribute radial coordinates with density $\rho(r)=\alpha e^{\alpha(r-R)}$, where $\alpha=(\gamma-1) / 2$ 
and hyperbolic dist radius $R=2 \ln \left(\frac{N}{c}\right)$, where

$$
c=\bar{k} \frac{\sin (\pi T)}{2 T}\left(\frac{\gamma-2}{\gamma-1}\right)^{2},
$$

and $T$ controls clustering. Then, connect each pair of nodes $i$ and $j$ with probability

$$
p\left(x_{i j}\right)=\frac{1}{1+e^{\left(x_{i j}-R\right) / 2 T}},
$$

where $x_{i j}$ is the hyperbolic distance satisfying

$$
\cosh x_{i j}=\cosh r_{i} \cosh r_{j}-\sinh r_{i} \sinh r_{j} \cos \Delta \theta_{i j} .
$$

It can be proved that the generated graph follows power-law distribution.

\subsection{Maximum likelihood estimation}

We fit the probability network to the model above by finding set of coordinates $\left\{r_{i}, \theta_{i}\right\}$ maximizing the likelihood that coordinates generated by the hyperbolic model match the probability network $\left\{p_{i j}\right\}$. This likelihood is

$$
\mathcal{L}\left(\left\{r_{i}, \theta_{i}\right\} \mid\left\{p_{i j}\right\}\right)=\frac{P\left(\left\{r_{i}, \theta_{i}\right\}\right) \mathcal{L}\left(\left\{p_{i j}\right\} \mid\left\{r_{i}, \theta_{i}\right\}\right)}{P\left(\left\{p_{i j}\right\}\right)},
$$

where

$$
P\left(\left\{r_{i}, \theta_{i}\right\}\right)=\frac{1}{(2 \pi)^{N}} \prod_{i=1}^{N} \rho\left(r_{i}\right)
$$

and

$$
\mathcal{L}\left(\left\{p_{i j}\right\} \mid\left\{r_{i}, \theta_{i}\right\}\right)=\prod_{i<j}\left[p\left(x_{i j}\right) p_{i j}+\left(1-p\left(x_{i j}\right)\right)\left(1-p_{i j}\right)\right] .
$$

Maximization of the likelihood in 1 is solved by the following Metropolis-Hastings algorithm.

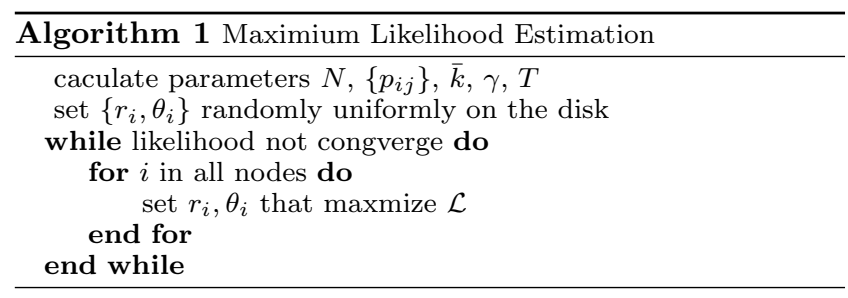

\subsection{Greedy forwarding}

In the greedy forwarding, nodes simply choose the next hop from neighbors which maximize the contact probability

$$
p=1+\frac{\lambda_{2}}{\lambda_{1}-\lambda_{2}} e^{-\lambda_{1}}+\frac{\lambda_{1}}{\lambda_{2}-\lambda_{1}} e^{-\lambda_{2}},
$$

where $\lambda_{1}$ denotes intensity of encounter process from the current node to the next node, and $\lambda_{2}$ from the next node to the destination node which can be derived from $p\left(x_{i j}\right)$ [7].

\section{RESULTS}

We use two real-world human mobile traces of Reality for the performance evaluation. We show the mapping result of Reality in Fig. 2, where different colors represent the contact frequency. The result show that there are two big mobile communities and several small ones in Reality. Node pairs that interact often are mapped close to each other on the hyperbolic plane, with hot nodes located near the center. If using the greedy algorithm to forward message from
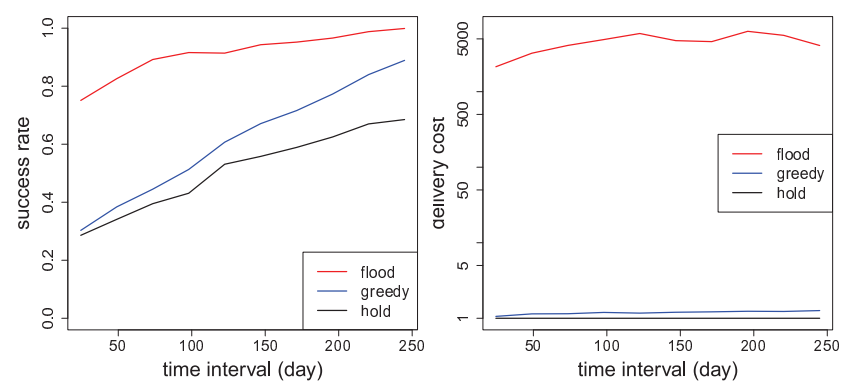

Figure 3: Forwarding results of Reality

one node to another, the forward path naturally follows popular node at local community - popular node at the destination's community - destination sequence, which is similar with BUBBEL Rap. Thanks to the huge space of the hyperbolic plane, we are able to locate nodes almost uniformly (in hyperbolic space). Thus, greedy forwarding is possible.

Fig. 3 shows the forwarding results, where 1,000 messages with random source and destination are generated randomly for each time interval. The success ratio stands for the proportion of the messages that are sent to the destination within specified time interval. Meanwhile, the delivery cost stands for the average number of copies generated during the forwarding process. Hold algorithm stores the message until source node meet destination node, which represents the lower bound of delivery cost. Flood algorithm flood message to every node encountered, which represents the upper bound of success ratio. The results show that our algorithm performs well with few forwarding cost, especially when time interval increases.

\section{REFERENCES}

[1] Y. Li, G. Su, et al., "The impact of node selfishness on multicasting in delay tolerant networks," IEEE Transactions on Vehicular Technology, vol. 60, no. 5, pp. 2224-2238, 2011.

[2] Y. Li, P. Hui, et al., "Evaluating the impact of social selfishness on the epidemic routing in delay tolerant networks," IEEE Communications Letters, vol. 14, no. 11, pp. 1026-1028, 2010.

[3] P. Hui, J. Crowcroft, and E. Yoneki. BUBBLE rap: Social-based forwarding in delay-tolerant networks. IEEE Trans. on Mobile Computing, 10(11):1576 -1589, Nov. 2011.

[4] Y. Li, Y. Jiang, et al., "Energy-efficient optimal opportunistic forwarding for delay-tolerant networks," IEEE Transactions on Vehicular Technology, vol. 59, no. 9, pp. 4500-4512, 2010.

[5] Y. Li, Z. Wang, et al., "Collaborative vehicular content dissemination with directional antennas," IEEE Transactions on Wireless Communications, vol. 11, no. 4, pp. 1301-1306, 2012.

[6] M. Boguñá, et al., Sustaining the internet with hyperbolic mapping. Nat Commun, 1:62, Sept. 2010.

[7] Y. Li, D. Jin, et al., "Revealing contact interval patterns in large scale urban vehicular ad hoc networks," ACM SIGCOMM Computer Communication Review, vol. 42, no. 4, pp. 299-300, 2012. 